\title{
An Efficient Algorithm for Downconverting Multiple Bandpass Signals Using Bandpass Sampling
}

\author{
Ngai Wong and Tung-Sang Ng \\ Department of Electrical and Electronic Engineering, \\ The University of Hong Kong, \\ Pokfulam Road, Hong Kong. \\ nwong@eee.hku.hk, tsng@eee.hku.hk \\ Tel.: ++ $852+28578406$, Fax: ++852 + 25598738
}

\begin{abstract}
Bandpass sampling is a useful alternative for direct digital downconversion in software radio. It significantly relaxes the analog-to-digital converter (ADC) sampling rate requirement and facilitates the design goal of moving the $A D C$ as close as possible to the antenna. This paper presents a modified interpretation to the graph of allowable sampling frequencies in uniform bandpass sampling. It is shown that the position and guard bands of a downconverted bandpass signal band are highly related to the order of the valid sampling range, called wedge order. An efficient algorithm is then proposed, which significantly reduces the computational load in determining the valid sampling frequencies to downconvert multiple distinct bandpass signals. Conditions for the placement of bandpass signals to utilize a given sampled bandwidth are also discussed.
\end{abstract}

\section{INTRODUCTION}

A goal in software radio digital front-end design is to push the digitization point as close as possible to the antenna [1]. Subsequent operations on the digital signal are then performed in the "soft", reconfigurable digital domain via digital signal processors (DSPs) or field programmable gate arrays (FPGAs). Ideally, different air interfaces can co-exist on a common hardware platform. The desired channel and its corresponding demodulation are then selected and carried out through programming [2].

In practice, the traditional Nyquist rate sampling is not feasible due to the high carrier frequencies of those bandwidths of interest. For example, the Global Positioning System Standard Positioning Service (GPSSPS) uses the $L 1$ frequency of $1575.42 \mathrm{MHz}$ and a $2 \mathrm{MHz}$ null-to-null bandwidth [3]. Direct digitization at Nyquist rate necessitates a sampling frequency as high as about $3.2 \mathrm{GHz}$, a rate impractical for existing discrete time signal processing systems. Moreover, it would be a waste to process at this rate for an information bandwidth of the order of only $2 \mathrm{MHz}$.

Bandpass sampling offers an attractive alternative solution. It is a special form of undersampling that translates (aliases) a high frequency bandpass signal to a lowpass one near to the zero frequency [4], [5]. The sampling frequency requirement is based on the signal

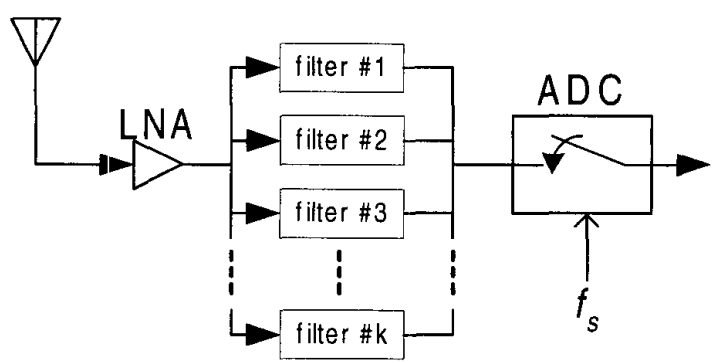

Fig. 1 . Block diagram of receiver front-end for $k$ distinct bandpass channels proposed by D. M. Akos et al. [6] for multi-band digital downconversion.

bandwidth rather than its highest frequency. The chief advantage of bandpass sampling, therefore, is the reduced requirement of the sampling frequency and of the associated digital processing capability. Power consumption, which is of importance to mobile devices, is alleviated by this lower processing rate. Also, the elimination of analogue mixers removes their corresponding non-idealities like DC offset, phase and gain mismatches etc. Nonetheless, bandpass sampling demands some critical hardware needs. First, the ADC analog input bandwidtlı must be able to "see" the bandpass input at its carrier frequency, probably at IF or RF. Second, narrow bandpass filters of very high $Q$ s are required to suppress the out-of-band noise. This is due to the fact that all noise within the analog input bandwidth of the ADC will be folded into the resulting lowpass band, called the sampled bandwidth.

To exploit the benefits of bandpass sampling, and due to the inadequacy of real time programmable processing power nowadays, D. M. Akos et al. [6] proposed a novel multi-band digitization method that utilizes bandpass sampling to significantly reduce the required ADC sampling frequency. The configuration of their proposed receiver front-end is shown in Fig. 1. The filters are narrow bandpass filters centered about multiple RF carriers of 


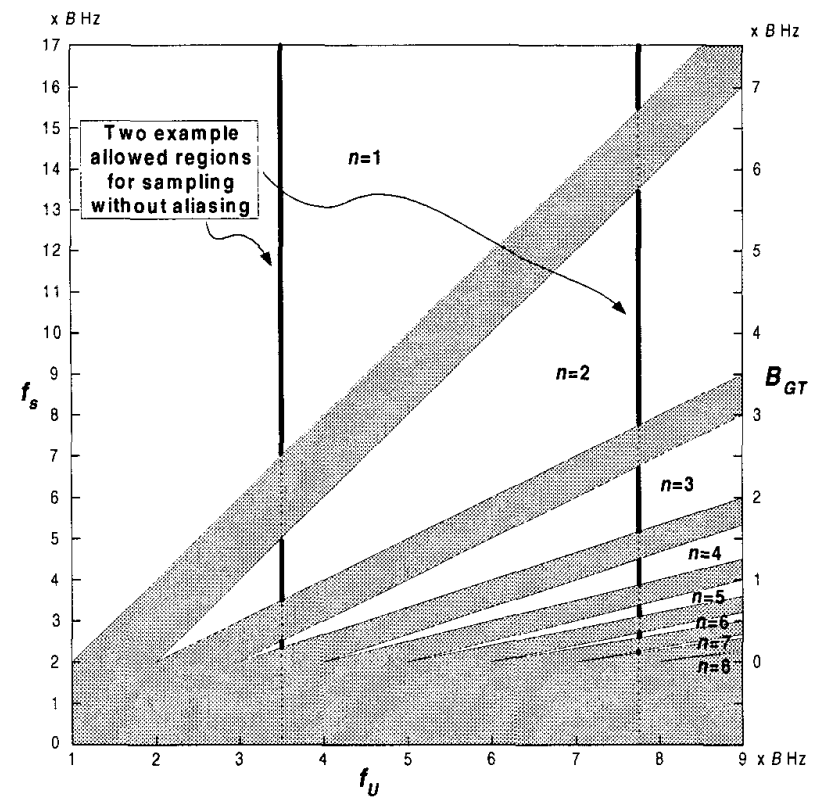

Fig. 2. Acceptable and unacceptable (shaded area) sampling frequencies for a bandpass signal of bandwidth $B$ located at $\left(f_{L}, f_{U}\right)$. Allowed ranges are shown in thickened dark lines for the examples of $f_{U}=3.5 \mathrm{~B} \mathrm{~Hz}$ and $f_{U}=7.75 \mathrm{~B}$ respectively.

interest. The appropriate sampling frequency, $f_{S}$, of the $\mathrm{ADC}$ is then determined such that all wanted bandpass signals are aliased into the sampled bandwidth $\left[0, f_{S} / 2\right]$ without causing aliasing. The non-linear nature of the sampling constraints of each signal, however, lends itself to computer simulation for the determination of an appropriate sampling frequency. A drawback is that an exhaustive testing of all frequencies up to the Nyquist rate, under the constraint of no aliasing, is computationally intensive. Since the procedure for sampling frequency determination was not further elaborated in [6] or in any other literature to date, this paper proposes a computationally efficient algorithm for this purpose based on a modified interpretation of the graph of allowable sampling frequencies in uniform bandpass sampling.

The paper is organized as follows. In Section II, the graph of allowable sampling frequencies for uniform bandpass sampling is reviewed and a modified interpretation is introduced. Section III proposes an efficient algorithm for determining an appropriate sampling frequency to simultaneously downconvert multiple bandpass signals without aliasing. Examples are given for verification of the algorithm's effectiveness. Section IV discusses bandpass signal placement conditions that optimize the available sampled bandwidth and conclusion is drawn in Section V.

\section{BANDPASS SAMPLING - THE GRAPHICAL APPROACH}

This section describes the bandpass sampling of a single bandpass signal and the next section extends it to multiple signals. For uniform sampling of a bandpass signal in the open interval $\left(f_{L}, f_{U}\right)$ with bandwidth $B=f_{U}-f_{L}$, the conditions for valid sampling frequencies are [4]

$$
\frac{2 f_{U}}{n} \leq f_{s} \leq \frac{2 f_{L}}{n-1}
$$

where $n$ is an integer given by

$$
1 \leq n \leq\left\lfloor f_{U} / B\right\rfloor \text {. }
$$

Here $\lfloor\circ\rfloor$ denotes the floor function. The plot of (1) and (2) is depicted in Fig. 2. The wedges represent allowed zones of sampling frequencies without causing aliasing. Obviously, the wedge of order 1 (i.e., $n=1$ ) represents the Nyquist sampling zone. For a bandpass signal of bandwidth $B$ and a band upper bound $f_{v}$ (e.g., $3.5 B \mathrm{~Hz}$ and $7.75 B \mathrm{~Hz}$ respectively in Fig. 2), the valid sampling frequency ranges are denoted by vertical lines above $f_{u}$ and within the wedges (shown by thickened line segments in Fig. 2). 


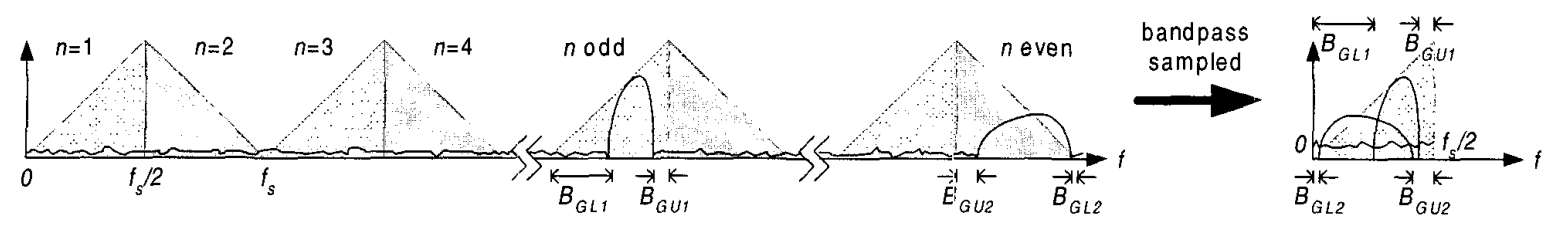

Fig. 3. Frequency domain representation of bandpass sampling using aliasing triangles.

The total guard band $B_{G T}$ is defined in this paper as

$$
B_{G T}=\frac{f_{S}}{2}-B
$$

Different from the definition in [4] where the guard bands are embedded into the signal band and treated as part of the signal bandwidth, the guard band definition in (3) represents the tolerance of the downconverted signal band from the boundaries of the sampled bandwidth $\left[0, f_{S} / 2\right]$. This is best illustrated by the concept of aliasing triangles (e.g., see [6]) as shown in Fig. 3. Here the allowances on both sides of the signal band in the sampled bandwidth add up to the total guard band. Additionally, it can be seen that all noise within the analog input bandwidth of the ADC is folded into the sampled bandwidth, therefore the requirement of steep roll-off narrow bandpass filters to maintain a reasonable signal-tonoise ratio (SNR).

A careful analysis of (1), (2) and Fig. 3 reveals an important fact. Specifically, the wedge order $n$ in Fig. 2 is equivalent to the triangle order in Fig. 3. In other words, valid frequencies falling in the $n$th wedge represent those sampling frequencies such that the bandpass signal will reside in the $n$th aliasing triangle. Using this idea, the relation between the guard bands and wedge order can be derived in accordance with Fig. 4. First, the span of allowable sampling frequencies $\Delta f_{s}\left(=\Delta f_{S L}+\Delta f_{S L}\right)$ is

$$
\Delta f_{S}=\frac{2\left(f_{U}-n B\right)}{n(n-1)}
$$

which decreases with increasing $n$. By breaking down the total guard band $B_{G T}$ into upper $\left(B_{G U}\right)$ and lower $\left(B_{G L}\right)$ guard bands, i.e., $B_{G T}=B_{G U}+B_{G L}$, it can be verified that for an odd $n$,

$$
B_{G U}=\frac{n}{2} \Delta f_{S L}
$$

while for an even $n$,

$$
B_{G U}=\frac{f_{U}-n B}{n}-\frac{n-1}{2} \Delta f_{S L} .
$$

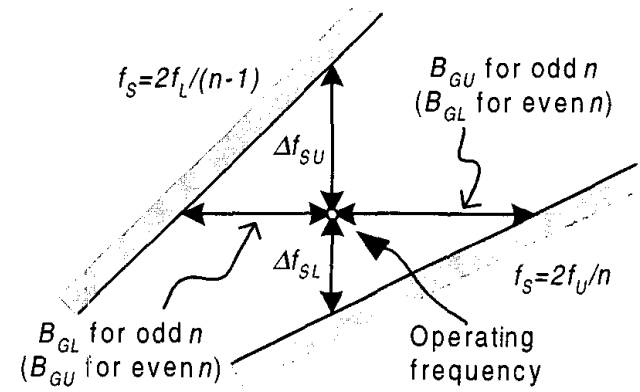

Fig. 4. Expanded view of the $n$th wedge in Fig. 2 .

Equations (4)-(6) infer that in an odd order wedge, when the sampling frequency is increased, the resultant signal band in the sampled bandwidth will move further apart from the upper bound of $f_{s} / 2$ until it touches zero, while the opposite happens for an even order wedge. Fig. 5 depicts such scenario, again for the example of bandpass signals of bandwidth $B$ with $f_{v}=3.5 B \mathrm{~Hz}$ and $7.75 \mathrm{~B} \mathrm{~Hz}$ respectivelly.

Several observations can be made from Fig. 5. First, the bandpass sampled signal band appears in the form of parallelogram stripes in the sampled bandwidth against $f_{s}$, with the vertical stripe height being the signal bandwidth $B$. The number of band stripes corresponds to the number of wedges that the vertical line cuts through (see Fig. 2). A higher-order wedge results in a "shorter" stripe of lower sampling frequencies to the left of Fig. 5. With the exception of the Nyquist stripe, which is an infinite-length horizontal rectangle, all other stripes form a zigzag pattern. Odd order stripes are in the form of backslashes "I"s, while even order" stripes are in the form of slashes "/"s. The conditions of (1)-(6) guarantee that all stripes, within their own valid ranges, will just touch the lower and upper bounds of the sampled bandwidth with no bending (i.e., aliasing).

It should be stressed that for even order wedges, the bandpass sampled lowpass signal band is flipped about its 


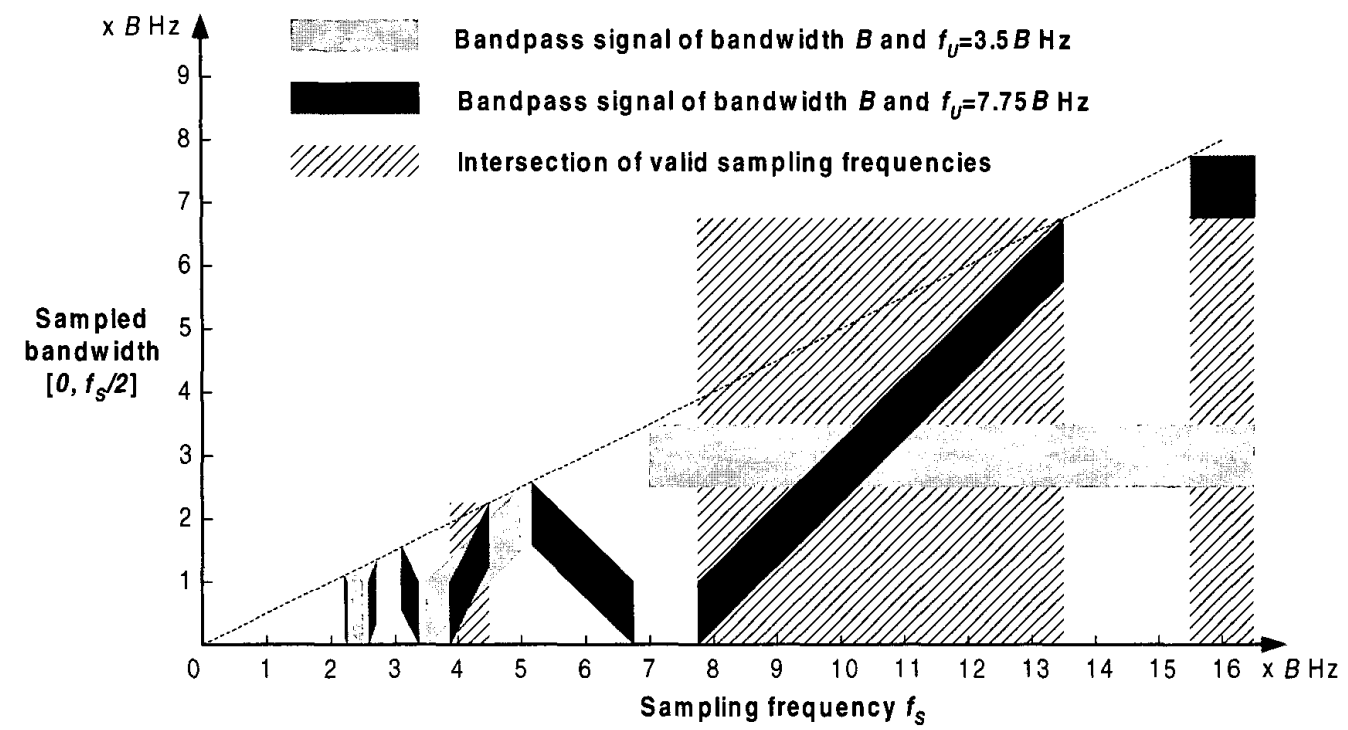

Fig. 5. Resultant signal band in the sampled bandwidth Vs sampling frequency.

center (see Fig. 3) and should be handled with care. This effect does not affect symmetric bandpass signal but for asymmetric signals, e.g., single-sideband (SSB) signals, this flipping should be accounted for in the digital signal processing part.

\section{DOWNCONVERTING MULTIPLE BANDPASS SIGNALS}

To fulfil the software radio philosophy of accommodating multiple standards, the results from Section II can be extended easily to downconvert multiple distinct, nonadjacent bandpass signals. The essence lies in the determination of a sampling frequency lower than the Nyquist rate that will translate all bandpass signals into the sampled bandwidth without causing aliasing, neither the aliasing of a signal onto its own nor into another. The algorithm is as follows,

1. The valid frequency ranges are computed for each bandpass signal using (1) and (2);

2. These sets of ranges are ANDed together to find their intersection;

3. The band stripes within these intersected ranges, knowing their wedge order, can be immediately obtained using the simple "stripe rule" from Section II;

4. The ranges where any two band stripes overlap are rejected. The remaining ranges represent the valid sampling frequencies for simultaneous downconversion of all bandpass signals without aliasing;
5. Among these valid frequency ranges, a desirable sampling frequency is chosen subject to constraints like minimum sampling frequency and/or other practical issues like sampling and carrier frequency drifts [7].

This algorithm is reflected in the example of Fig. 5. Instead of the brute force approach of testing all frequencies starting from the sum of bandwidths $(2 B \mathrm{~Hz})$ up to the Nyquist frequency $(15.5 B \mathrm{~Hz})$, only the intersection of the two sets of valid frequency ranges (represented by slant-line background) is tested. Apparently, the saving will increase further with the number of bandpass signals, as the intersection will be less. An exceptional case in step 4 is in the downconversion of Code Division Multiple Access (CDMA) signals where signal bands are allowed to overlap on one another provided a degradation in SNR is tolerable [6].

To get a better feeling of the effectiveness of the algorithm, the example from [6] is taken, in which the U.S. GPS-SPS ( $\left.f_{U}=1577.02 \mathrm{MHz}, B=3.2 \mathrm{MHz}\right)$ and the Russian Global Navigation Satellite System (GLONASS) ( $f_{L}=1609.40625 \mathrm{MHz}, B=7.5 \mathrm{MHz}$ ) were considered and sampling frequencies from $20 \mathrm{MHz}$ to $40 \mathrm{MHz}$ were tested. Using the proposed approach in this paper, it can be found that only an aggregate of $7.64 \mathrm{MHz}$ out of the $20 \mathrm{MHz}$ span needs to be tested. This represents a saving of $61.8 \%$. The stripe rule also provides a quick way to position the signal bands above a valid frequency range without having to 


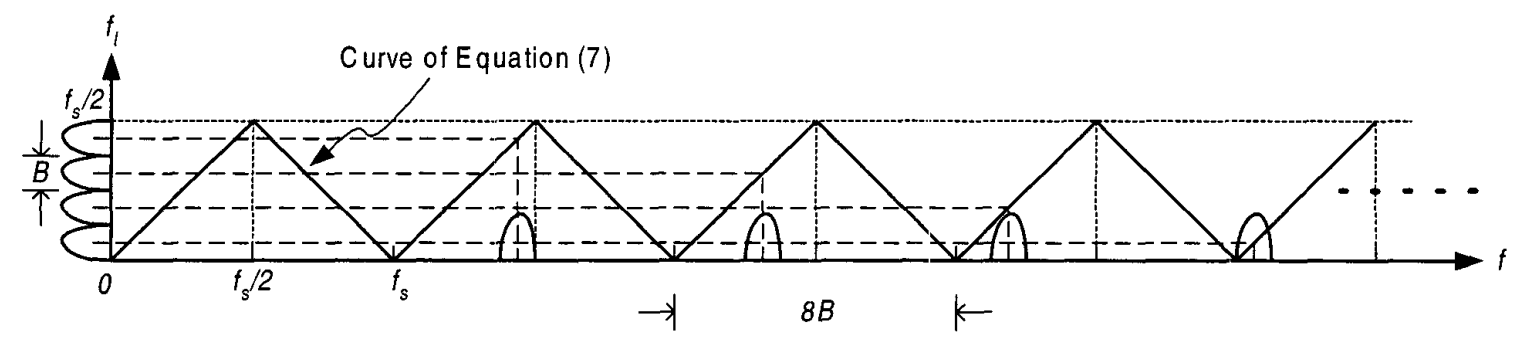

Fig. 6. Plot of (7) and an example sampled bandwidth optimization scheme for four ec|ually spaced channels of bandwidth $B$.

compute their location at every single frequency point, thereby further reduces the computational complexity.

\section{SIGNAL PLACEMENT UTILIZING SAMPLED BANDWIDTH}

A question may now arise as to what are the conditions for the placement of the multiple bandpass signals such that the sampled bandwidth can be fully utilized, i.e., the sampled bandwidth is exactly equal to the sum of signal bandwidths. Or suppose a sampling frequency is fixed for a certain system, in what way those bandpass signals can be positioned in order to utilize the sampled bandwidth. These questions are answered by considering the equation relating an arbitrary frequency $f$ and its image $f$ in the sampled bandwidth when being sampled by a frequency $f_{s}$, namely [6],

$$
f_{l}= \begin{cases}\operatorname{rem}\left(f, f_{s}\right) & \text { if } \operatorname{rem}\left(f, f_{s}\right) \leq f_{s} / 2 \\ f_{s}-\operatorname{rem}\left(f, f_{s}\right) & \text { if } \operatorname{rem}\left(f, f_{s}\right)>f_{s} / 2 .\end{cases}
$$

Here $\operatorname{rem}\left(f, f_{s}\right)$ is the remainder after division of $f$ by $f_{s}$. Equation (7) can be verified from Fig. 3 graphically. The plot of (7) is shown in Fig. 6 along with an example of positioning four equally spaced channels, all of bandwidth $B$. It can be seen that bandpass signal bands on the frequency axis are "reflected" back to the sampled bandwidth according to (7). Note that the image of a bandpass signal reflected by a negative slope will be flipped about its center in the sampled bandwidth, and a signal band traversing integral multiples of $f_{S} / 2$ will bring about aliasing, which are consistent with Fig. 3. With regard to (7) and Fig. 6, it can now be deduced that the sampled bandwidth is utilized when and only when the bandpass signals are positioned in such a way that their "reflections" (not their exact band locations) just add up to the sampled bandwidth without causing aliasing.

\section{CONCLUSION}

This paper has presented a modified interpretation to the graph of allowable sampling frequencies for bandpass sampling. Particular attention has been paid to the relation between the wedge order and the resultant band position and guard bands in the sampled bandwidth. A computationally efficient algorithm has been proposed for determining the appropriate sampling frequency to simultaneously downconvert multiple distinct bandpass signals. Numerical examples have been given to verify the effectiveness of the algorithm. Finally, conditions for the placement of bandpass signals to utilize a given sampling frequency have been elaborated.

\section{REFERENCES}

[1] IEEE Commun. Mag., vol. 33, pp. 26-68, May 1995.

[2] T. Hentschel, M. Henker, and G. Fettweis, "The digital front-end of software radio terminals," IEEE Personal Communications, vol. 6, no. 4, pp. 40-46, Aug. 1999.

[3] U.S. Department of Transportation, Global Positioning Systern Standard Positioning Service Signal Specification, June 2, 1995.

[4] R. G. Vaughan, N. L. Scott, and D. R. White, "The theory of bandpass sampling," IEEE Trans. Signal Processing., vol. 39, no. 9, pp. 1973-1984, Sep. 1991.

[5] H. P. Hsu, "Sampling," The Communications Handbook, Editor-in-Chief, J. D. Gibson. Boca Raton, Fla: CRC Press, pp. 13-22, 1997.

[6] D. M. Akos, M. Stockmaster, J. B. Y. Tsui, and J. Caschera, "Direct bandpass sampling of multiple distinct RF signals," IEEE Trans. Commun., vol. 47, no. 7, pp. 983-988, Jul. 1999.

[7] R. Qi, F. P. Coakley, and B. G. Evans, "Practical consideration for bandpass sampling," Electronics Letters, vol. 32,, no. 20, pp. 1861-1862, Sept. 1996. 